Economía, Sociedad y Territorio, vol. IV, núm. 16, 2004, 697-721

\title{
Productividad total de factores y reducción de costos en la industria manufacturera mexicana, 1994-1999
}

\author{
Armando Nevárez SidA* \\ Ángel Mauricio Reyes Terrón***
}

\begin{abstract}
The article analyzes total factor productivity in the mexican manufacturing industry in the period from 1994 to 1999. Applying the methodology proposed by Harberger (1998), the change in this variable is interpreted as a reduction or a real increment of costs. It was possible to identify both those subsectors and industrial classes that reduced or increment costs and the magnitude of these changes in monetary terms. In general, significant reduction of costs within a subsector of an industrial class was rare, while there were minor reductions or increments in the other classes.
\end{abstract}

Keywords: Total factor productivity, real reduction of costs, economic growth.

\section{Resumen}

El presente trabajo analiza la productividad total de factores en la industria manufacturera mexicana en el periodo de 1994 a 1999. El cambio experimentado por esta variable es interpretado como reducción o incremento real de costos de acuerdo con la metodología propuesta por Harberger (1998). Se logró la identificación de aquellos subsectores y clases industriales que redujeron o aumentaron costos, así como la magnitud de estos cambios en términos monetarios. Las clases industriales que presentan una reducción de costos significativa al interior de un subsector son generalmente pocas, mientras que el resto presenta pequeñas reducciones de costos o incrementos.

Palabras clave: Productividad total de factores, reducción real de costos, crecimiento económico.

* Centro de Investigación y Docencia Económicas, México. Correo-e: armando.nevarez@cide.edu

** Centro de Investigación y Docencia Económicas, México. Correo-e: angel.reyes@cide.edu 


\section{Introducción $^{1}$}

Esta investigación versa sobre la industria manufacturera mexicana, y muestra una estimación de la productividad total de factores (PTF) de las diferentes clases industriales que integran los nueve subsectores en los que se divide la industria manufacturera en México. El estudio se circunscribe al periodo 1994-1999. La información utilizada para determinar la PTF proviene de los censos industriales correspondientes a esos años. Una vez determinada esta variable, su interpretación se efectúa aplicando la metodología propuesta por Harberger (1998), quien se disitingue de otros autores que le preceden en el estudio de la $\operatorname{PTF}^{2}$ debido a que propone explicar sus cambios como aumentos o disminuciones de costos.

Estudiar a la industria manufacturera mexicana reviste gran relevancia por varias razones. En primer término, porque buena parte del desarrollo nacional ha sido logrado gracias al impulso y contribución de este sector económico. En segundo lugar, las manufacturas han experimentado cambios significativos a lo largo de las últimas décadas, pues han tenido que adaptarse a las transformaciones impuestas por el cambio de rumbo del modelo económico.

La exposición de las empresas a procesos de apertura las ha obligado a mejorar su competitividad en todos sentidos. Así, en el seno de la industria manufacturera no sólo se llevan a cabo transformaciones significativas en materia de inversiones, sino también en materia de cambio tecnológico, innovación, desarro-

\footnotetext{
${ }^{1}$ Esta investigación se realizó como parte de las actividades del Programa de Ciencia y Tecnología del Centro de Investigación y Docencia Económicas, A.c. (México). Los autores desean agradecer la supervisión y valiosos comentarios del profesor Carlos Bazdresch Parada en la realización de este documento. Los errores u omisiones son responsabilidad exclusiva de los firmantes. Una versión previa de lo que aquí se presenta puede consultarse en el documento de trabajo núm. 210, CIDE, división de Economía, México, 2001.

${ }^{2}$ En una nota sobre este componente, Z. Griliches (1996) recupera el origen e historia del estudio de la PTF. Por su parte, otros autores han hecho esfuerzos por concentrar las diferentes formas de medir la PTF y esclarecer su interpretación teórica, por ejemplo: Nadiri (1970) plateó que el cambio en la productividad es la causa y a la vez la consecuencia de la evolución de fuerzas que dinámicamente operan de manera franca en una economía, algunas de ellas son intangibles y sólo pueden ser capturadas por el residuo; Prescott (1998) enfatiza en la necesidad de consolidar una teoría sobre la PTF y Hulten (2000) retoma la discusión teórica revisando las diferentes posturas, métodos y supuestos existentes, establece que la PTF se refiere a diversos componentes entre los que se encuentran los efectos del cambio tecnológico y organizacional así como elementos poco deseables como errores de medición, variables omitidas, problemas de agregación y especificación, etc.
} 
llo de productos y procesos. Además, por el lado del factor humano se dan transformaciones cualitativas orientadas al entrenamiento y capacitación, para aprovechar la experiencia y capacidad de la fuerza laboral a través de la acumulación de conocimientos, habilidades y aprendizajes.

La necesidad de conocer aquellos aspectos sobre la evolución reciente de la industria manufacturera mexicana y, en particular, los elementos que se asocian a su crecimiento que no están directamente relacionados con la productividad individual de cada uno de sus insumos productivos obligan a efectuar mediciones de la PTF a efecto de observar el comportamiento de este componente y estar en posibilidades de explicar su posible causalidad. Este documento ofrece evidencia empírica sobre el comportamiento de los subsectores y clases industriales de la industria manufacturera durante el periodo de análisis, y avanza en una línea de trabajo que no ha sido suficientemente explorada y que revela aspectos novedosos sobre el sector.

Los objetivos de este documento son: obtener un estimado de la PTF de la industria manufacturera mexicana entre 1994 y 1999, y presentar una cuantificación de la PTF como reducción real de costos para el caso de la industria manufacturera mexicana. Generada esta información, podemos estar en posibilidades de comparar e identificar, para el periodo seleccionado, aquellas clases industriales que reducen o aumentan costos.

\section{Antecedentes}

En general, la economía ha considerado que el crecimiento económico, particularmente del producto, se debe al incremento de los insumos o factores de producción; sin embargo, esto no es necesariamente cierto puesto que en el crecimiento de la producción intervienen otros elementos que no son capturados fielmente por los componentes tradicionales: trabajo y capital.

La presencia de un término de error o residuo en las estimaciones del growth accounting no es casual. Se asume que dicho remanente representa todos aquellos elementos no explícitamente incorporados en la función de producción y, para sorpresa de muchos, en ocasiones este residuo alcanza dimensiones superiores a las que reportan los factores de producción. Esto significa que muchas veces, lo no explicado es significativamente más grande que lo explicado por los stocks de trabajo y capital. 
La complementariedad esencial entre la productividad del trabajo y del capital en el producto ha llevado a cuantificar el peso de ambos componentes en conjunto, con el propósito de encontrar una medida de la PTF que hace referencia al crecimiento del residuo y que intenta explicarlo. ${ }^{3}$ Así, se ha planteado la necesidad de recuperar los diferenciales de la remuneración a los factores entre empresas e industria a efecto de establecer evidencia que permita sostener interpretaciones sobre el residuo como aquellas que lo vinculan al cambio tecnológico, a mejoras en la propia PTF y a la reducción real de costos unitarios.

El problema con la explicación del residuo como cambio tecnológico es su limitada concreción, pues como el propio R. M. Solow (1957) refirió, el cambio tecnológico puede ser cualquier cosa que origine modificaciones en la función de producción como mejoras en la calidad de los insumos trabajo y capital; sin embargo, puede tratarse de muchas cosas a la vez, como invenciones, gasto en actividades de investigación y desarrollo (IyD), ${ }^{4}$ innovaciones de todo tipo, etc. La idea del cambio tecnológico se distingue por ser relativamente impalpable. Por su parte, la postura de que el residuo expresa 'mejoras en la PTF' alude a posibles externalidades de cualquier tipo que inciden sobre los movimientos y cambios del propio residuo, por ejemplo: economías de escala, spillovers, ${ }^{5}$ etc.

\footnotetext{
${ }^{3}$ Sargent, T. C. et al. (2000) abordan el debate existente entre quienes privilegian el uso de la PTF como medida de crecimiento versus aquellos que conceden mayor importancia a la productividad del trabajo. Señalan que ambas ofrecen ventajas y desventajas, de ahí que sea necesario seleccionar en qué momento utilizarlas según el periodo analizado, la calidad de los datos sobre los stocks de capital y el modelo de crecimiento considerado.

${ }^{4}$ En México, algunas líneas de investigación se han enfocado hacia diversas variables que contribuyen a explicar la conducta innovadora del sector manufacturero; así se ha avanzado en diversas direcciones, como el gasto en IyD tecnológico, la disponibilidad de capital humano y la corrección de su escasez sobre todo en áreas técnicas. Estos trabajos no se refieren directamente al crecimiento de la PTF pero avanzan algunas hipótesis relacionadas con su crecimiento. Sobresalen: el trabajo de Meza y Mora (2002), que expone evidencia sobre los determinantes del gasto de inversión en IyD en las empresas manufactureras mexicanas y los de López-Acevedo (2002a, 2002b y 2002c), entre otros.

${ }^{5}$ Por ejemplo: Grether, J. M. (1999) trata de extender el análisis de las derramas (spillovers) tecnológicas generadas por la inversión extranjera directa en el periodo 19841990, que corresponde a una de las primeras fases del proceso de apertura comercial de México. Sus resultados indican que el capital extranjero y la estructura del mercado se relacionan positivamente con la eficiencia productiva a nivel de planta, pero no a nivel de industrias, lo que sugiere que las empresas eficientes ganan participación en el mercado, pero la tecnología no se difunde libremente entre sectores. Adicionalmente, la difusión de tecnología es favorecida por el tamaño de la planta y por las economías de aglomeración, pero no por la mayor exposición a mercados internacionales durante ese periodo.
} 
La versión de que el residuo puede ser visto como 'reducción real de costos' no tiene el ánimo de identificarlo con una nueva etiqueta, pero sí trata de interpretarlo de una manera diferente. Esta postura parece estar más en línea con la visión de directivos y estrategas en producción de una empresa o corporación. $\mathrm{Mu}-$ chas empresas persiguen directamente el objetivo de ser competitivas en el mercado por la vía de reducir sus costos de producción. Costos bajos tienen la ventaja de mejorar la rentabilidad de las firmas en etapas de auge, pero también permiten contar con un medio de defensa ante choques económicos o recesiones. Aquí surge el siguiente cuestionamiento: ¿cómo reducir costos al interior de una empresa? La respuesta puede ser muy amplia, ya que pueden existir muchas maneras de bajar los costos manteniendo constantes los rendimientos, o mejorándolos.

El abatimiento de costos puede ocurrir a partir de una mayor eficiencia en el uso de los recursos, nuevas técnicas productivas, cambios organizacionales, mejoras en las redes de comunicación e intercambio de información entre los miembros de la empresa, procesos de aprendizaje, fusiones y acuerdos de cooperación con otros agentes económicos, economías de escala, etc.

La postura anterior permite separar aquellos aumentos del producto obtenido que provienen de los stocks de factores trabajo y capital de aquellos que surgen de otras posibles fuentes y que pueden ser muchas y muy diversas. Harberger (1998) plantea que las causas del crecimiento no siempre son detectadas por las herramientas de medición tradicionales y sugiere que hay mil formas de reducir los costos. La reducción de costos que experimenta una empresa o una industria tiene una esencia compleja y multifactorial, que puede ser observable al cabo de varios años y puede resumirse a través de una sola medida.

Harberger (1998) afirma que la interpretación del crecimiento del residuo como 'reducción real de costos' le da sentido a dicho componente, pues lo vuelve una magnitud tangible en términos monetarios. Esto vincula al residuo con la empresa, sus directivos y personal de producción. Así, el residuo puede describir el abatimiento de costos unitarios reales durante un periodo en una empresa o industria.

El mismo autor plantea que el proceso de crecimiento probablemente no es como se ha descrito hasta ahora y lo compara con lo que ocurre entre yeasts vs. mushrooms. Señala que "un proceso yeasts (levadura) hace que el pan se expanda como un balón que se llena de aire”. Trasladando esta visión a la realidad, se 
trata de aquella situación generada por externalidades o economías de escala que causan efectos sobre toda la economía. En cambio, "un proceso mushrooms (hongos) ocurre ya que éstos surgen de manera espontánea y poco predecible”, así, los mushrooms representan avances en cuanto a PTF (o reducción real de costos) pudiendo deberse a mil y un causas, pues aparecen en forma irregular, habitualmente en grupos (clusters), y pueden ocurrir más en unas industrias en comparación con otras.

Para Harberger, el crecimiento ocurre más como un proceso mushrooms y advierte que el resultado de las perturbaciones comunes es despreciable en comparación con aquellas que causan cambios en la productividad y que son más específicas de cada industria.

\section{Datos y metodología}

La información que se utilizó para llevar a cabo esta investigación proviene de los censos industriales que realiza y publica el Instituto Nacional de Estadística, Geografía e Infrormática (INEGI) en forma periódica, en particular se utilizaron datos para 1994 y 1999.

La información censal disponible permitió medir la concentración del crecimiento de la productividad total de los factores para los subsectores del sector manufacturero que son: 31 Producción de alimentos, bebidas y tabaco; 32 Producción de textiles, prendas de vestir e industrias del cuero; 33 Manufacturas de la madera; 34 Producción de papel, productos de papel, imprentas y editoriales; 35 Producción de sustancias químicas y artículos de plástico o hule; 36 Producción de bienes con base en minerales no metálicos; 37 Industrias metálicas básicas; 38 Manufacturas de productos metálicos, maquinaria y equipo; 39 Otras industrias manufactureras.

El presente documento aplica la metodología sugerida por Arnold C. Harberger (1998) que parte de la siguiente ecuación:

$$
\bar{p} \Delta y=\bar{w} \Delta L+(\bar{\rho}+\delta) \Delta K+R
$$

Donde:

$$
\begin{aligned}
& \Delta \\
& \text { ' } \mathrm{L}=\text { Cambio en el insumo trabajo; } \\
& \text { = Nivel general de precios inicial; }
\end{aligned}
$$


$=$ Salario real inicial;

$\bar{\rho}=$ Tasa de rendimiento del capital real inicial;

= Tasa de depreciación real inicial;

' $\mathrm{K}$ = Cambio en el stock de capital;

$\mathrm{R}=$ 'Residuo' o crecimiento no explicado por el incremento en los insumos tradicionales.

$\mathrm{O}$ alternativamente:

El residuo representa una fracción importante del crecimiento del producto. De la discusión teórica sobre la explicación de la PTF sabemos que ha estado centrada en el 'capital humano' y el 'avance tecnológico'. Sin embargo, actualmente se considera que la PTF expresa cambio tecnológico, reducción real de costos o mejoras en la propia PTF. ${ }^{6}$ En particular, Harberger propone ob-

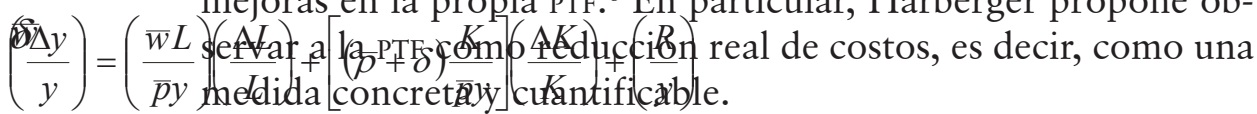

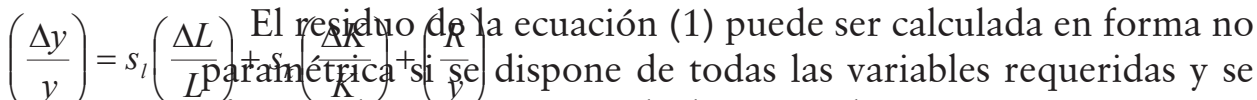
efectúan las operaciones algebraicas; alternativamente, se pueden emplear métodos paramétricos en cuyo caso las estimaciones pueden capturar errores de medición (OECD, 2001a y 2001b). En el presente estudio, dadas las limitaciones impuestas por los datos censales, se recurrió al segundo procedimiento de acuerdo con la ecuación (1') planteada por Harberger.

Una vez determinada la PTF como residuo, se procedió a tabular la información. La PTF es transformada en reducción o aumento de costos para cada industria. Finalmente, a partir de las tabulaciones los datos deben ordenarse en forma tal que permitan ser graficados como curva de Lorenz.

\footnotetext{
${ }^{6}$ En un estudio sobre México, el Banco Mundial (1998) trata la dinámica y evolución de la PTF, haciendo hincapié en el marco institucional y la necesidad de avanzar reformas estructurales que influyan sobre los mercados y permitan transmitir información e incentivos institucionales a los agentes económicos encausándolos hacia mercados formales. El documento se encamina hacia la identificación de posibles recomendaciones de política con el propósito de mejorar el desempeño productivo de México.
} 


\section{Resultados}

La reducción o incremento real de costos que presentan las diferentes agregaciones de la industria manufacturera para el periodo 1994-1999 es analizada por subsectores descendiendo a un nivel de clase industrial. Es importante precisar que existen subsectores que registran ascenso de costos, aunque algunas de las clases que los constituyen presenten una reducción efectiva de los mismos durante el periodo.

\subsection{Subsectores con reducción de costos}

A continuación se presentan, a título de ejemplo, algunos de los resultados obtenidos para el subsector 33: Manufacturas de la madera, que como saldo del periodo presenta reducción real de costos (véase el cuadro 1).

La primera columna nos indica las clases industriales que componen el subsector 33. La segunda columna corresponde al crecimiento de la productividad total de factores, y puede interpretarse como un porcentaje $(1=100)$ de reducción o aumento de costos; desde luego, las clases que presentan valores positivos tuvieron una reducción real de costos y viceversa.

La tercera columna indica el valor de la reducción real de costos en millones de pesos de 1993, y resulta del producto de la PTF por el valor agregado inicial; expresa en términos monetarios la magnitud aproximada de la disminución o crecimiento de costos resultante al final del periodo. ${ }^{7}$ La columna cuatro es el percentil de la reducción efectiva de costos, y ha sido calculada como porcentaje acumulado de la caída de costos respecto de todo el subsector en su conjunto; por ejemplo, a la primer clase industrial de la tabla (fabricación y reparación de persianas) se le puede atribuir el 35.9 por ciento de la reducción real de costos del subsector 33. El dato del valor agregado inicial proviene directamente del censo industrial de 1994. Finalmente, la columna restante constituye el percentil del valor agregado inicial que no es más que el porcentaje acumulado del valor agregado de cada una de las clases industriales, es decir, las dos primeras clases industriales

${ }^{7}$ La reducción o el aumento de costos es, en algunos casos, mayor al valor agregado inicial, pero su magnitud es inferior a los ingresos provenientes de la actividad, por lo que no es relevante dado que la reducción o aumentos de costos es el acumulado durante el periodo. 


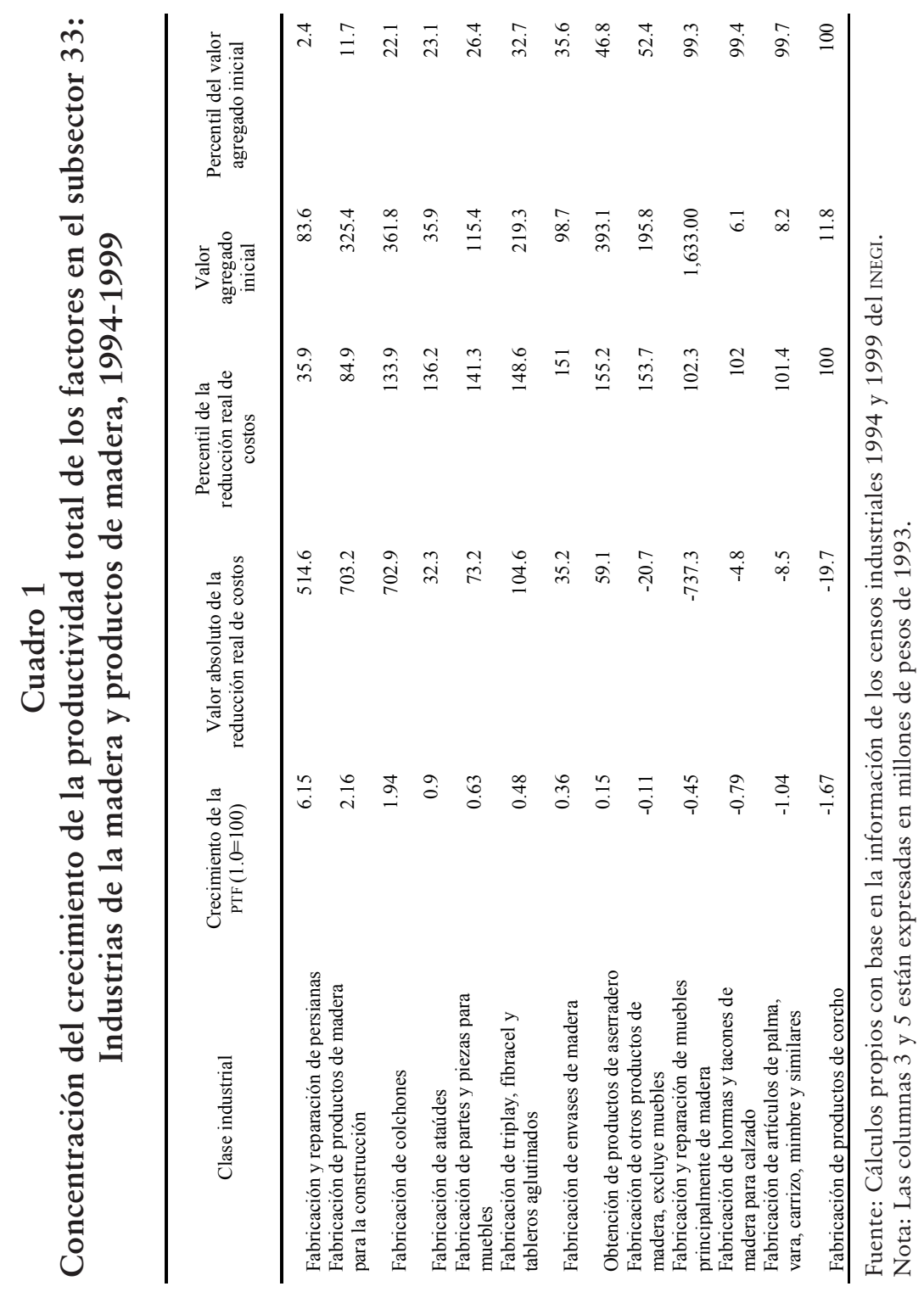


presentadas en el cuadro participaron con 11.7 por ciento del valor agregado inicial en este subsector para el año de 1994.

En resumen, la clase industrial correspondiente a la fabricación y reparación de persianas participó con un 2.4 por ciento del valor agregado del subsector manufacturas de la madera en 1993, con una reducción real de costos de 514.6 millones de pesos de 1993 durante el periodo 1994-1999.

La clase industrial de fabricación y reparación de muebles principalmente de madera experimentó entre 1994 y 1999 una PTF de -0.45 , lo que implica un aumento de costos de 737.3 millones de pesos de 1993. Como puede observarse, el valor agregado de esta clase de actividad fue de 1,633 millones de pesos en el periodo de inicio. Esta clase industrial es relevante porque representa 46.9 por ciento del valor agregado total del subsector 33, asimismo explica más del 50 por ciento de la reducción real de costos del subsector de manufacturas de la madera.

En la gráfica 1 se presentan los datos del cuadro anterior en un formato cercano a la curva de Lorenz, ${ }^{8}$ en el eje ' $y$ ' se tiene el percentil de la reducción real de costos mientras que el eje ' $x$ ' representa el percentil del valor agregado inicial para las clases industriales que comprenden el subsector 33 de manufacturas de la madera. La forma como se ordenaron las clases industriales, tanto en la presentación tabular como gráfica, está en función del crecimiento de la PTF. Las clases industriales que redujeron más sus costos respecto de su valor añadido son las que se ubican en las primeras posiciones.

La interpretación de la gráfica es análoga a la de la curva de Lorenz: la línea de 45 grados nos indica que una industria o conjunto de industrias que participa con un determinado porcentaje del valor agregado inicial contribuyó dentro de la reducción de costos del subsector con el mismo porcentaje. Las tres primeras clases industriales presentadas en el gráfico: fabricación y reparación de persianas, fabricación de productos de madera para la construcción y fabricación de colchones fueron las que redujeron más los costos en este subsector.

Otras actividades que también tuvieron disminuciones de costos fueron: fabicación de ataúdes; fabricación de partes y piezas

\footnotetext{
${ }^{8}$ En mediciones de concentración industrial, es frecuente representar a la curva de Lorenz en forma diferente a la que se hace en el análisis de distribución del ingreso. El cambio consiste en ordenar las unidades acumuladas que se muestran en el eje de las abscisas de mayor a menor, en lugar de hacerlo de menor a mayor; esto implica que la curva se encuentre por encima de la línea de equidistribución en lugar de ubicarse por debajo (véase: Pablo, 1997).
} 


\section{Gráfica 1}

Subsector 33: Industrias de la madera y productos de madera

para muebles; fabricación de triplay, fabricel y tableros aglutinados; fabricación de envases de madera, y obtención de productos de aserradero. Estas clases industriales alcanzaron disminuciones de costos menos significativas que las descritas anteriormente.

Las clases industriales restantes ${ }^{9}$ representaron 53.2 por ciento de las actividades del subsector 33 y obtuvieron aumentos de costos; tales industrias son: fabricación de otros productos de madera, excluye muebles; fabricación y reparación de muebles principalmente de madera; fabricación de hormas y tacones de madera para calzado; fabricación de artículos de palma, vara, carrizo, mimbre y similares; fabricación de productos de corcho.

Siguiendo esta misma línea de interpretaciónmpuede decirse quê la reducción real de costos del subsector de manufacturas de la madera durante el periodo 1994-1999 está explicada por el 15 por ciento de tas clases industriales que la comprenden (medidas por el valor agregado inicial), representado por la primera línea

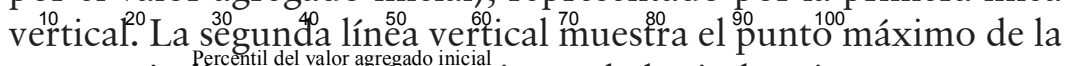
curva, e indica que 46.8 por ciento de las industrias que componen este subsector industrial presentaron reducciones de costos durante 1994-1999, mientras que 53.2 por ciento de las industrias restantes tuvieron un incremento real de costos.

El promedio ponderado de reducción de costos del sector 33 para el periodo 1994-1999 fue de 11.24 por ciento. El porcentaje de clases industriales con reducción real de costos con base en el valor agregado de 1993 fue de 82.4 por ciento, y el 17.6 por ciento sobrante experimentó movimientos alcistas de costos. La

\footnotetext{
${ }^{9}$ Gráficamente, todas las clases de actividad industrial que tuvieron aumento de costos aparecen con pendiente negativa.
} 
reducción real de costos promedio anual para el subsector de manufacturas de la madera fue de 2.35 por ciento.

Otros subsectores manufactureros que reportaron reducción real de costos fueron el 35: producción de sustancias químicas y artículos de plástico o hule, 36: producción de bienes con base en minerales no metálicos y 39: otras industrias manufactureras.

En el caso del subsector 35, algunas de las clases industriales que consignaron una reducción de costos significativa ${ }^{10}$ son: la refinación de petróleo y fabricación de productos petroquímicos básicos; ambas representan más de 30 por ciento del valor agregado inicial y más de 40 por ciento de los activos fijos netos de este subsector. La clase de actividad que tuvo una mayor reducción de costos con respecto a su valor agregado inicial fue la de fabricación de perfumes, cosméticos y similares.

Contrariamente, las clases industriales que presentaron aumentos de costos significativos fueron: fabricación de películas, placas y papel sensible para la fotografía; fabricación de artículos de plástico reforzado y fabricación de juguetes de plástico. Otra clase importante de este subsector es la fabricación de productos farmacéuticos que presentó una reducción real de costos.

El 93.9 por ciento de las industrias que conforman el subsector 35 experimentaron reducción efectiva de costos. Alrededor de 62 por ciento de las industrias explica la reducción de costos experimentada por el subsector durante el periodo, en cambio 6.1 por ciento tuvieron aumento de costos. Estos resultados son importantes si consideramos que el subsector 35 ha sido uno de los más dinámicos y ha estado sujeto a innovación permanente.

El subsector 36 presentó seis clases industriales con disminución significativa de costos; tales industrias fueron: fabricación de mosaicos, tubos, postes y similares, a base de cemento; fabricación de fibra de vidrio y sus productos; elaboración de yeso y sus productos; fabricación de ladrillos, tabiques, tejas de arcilla no refractaria; fabricación de abrasivos y fabricación de concreto premezclado. De éstas, la más importante en cuanto a valor agregado es la de fabricación de concreto.

En el extremo opuesto estuvieron las industrias que aumentaron costos en el subsector 36, y son las siguientes: alfarería y cerámica; fabricación de otros artículos de vidrio y cristal no especificados anteriormente; fabricación de productos de asbesto, cemento y fabricación de vidrio plano, liso y labrado. La primera

${ }^{10}$ Una clase industrial tiene una reducción de costos significativa si el crecimiento de la PTF es mayor a uno. 
de éstas (la de alfarería y cerámica), si bien no es muy intensiva en capital, sí es importante en el número de personas que emplea. Adicionalmente, 70.8 por ciento (15 clases) de las industrias de este subsector tuvieron baja de costos y las siete restantes observaron aumentos.

El porcentaje promedio ponderado de reducción de costos en este segmento de las manufacturas fue de 45.57 durante el periodo considerado. Otras industrias relevantes que comprende este subsector son las de fabricación de envases y ampolletas de vidrio y la fabricación de cemento hidráulico que sufrieron aumento y reducción de costos, respectivamente.

El comportamiento del subsector 39 (otras industrias manufactureras) fue semejante al de los subsectores manufactureros $35 \mathrm{y}$ 36. Las clases industriales que sufrieron alza de costos reales significativos fueron: fabricación de juguetes y fabricación de cierres de cremallera. Otras dos más experimentaron aumentos moderados de costos. Las clases que disminuyeron costos significativamente fueron: la correspondiente a acuñación de monedas, fabricación de joyas y orfebrería de oro y plata, y fabricación y ensamble de instrumentos musicales y sus partes. La clase más importante de este subsector es la fabricación de artículos y útiles para oficina que durante el periodo presentó un ligero incremento en costos. Casi 30 por ciento de las industrias de este subsector manufacturero explican el 100 por ciento de la disminución en costos, al mismo tiempo, 59.5 por ciento de las industrias tuvieron reducción de costos en forma innegable. El porcentaje medio de reducción de costos para el subsector de otras industrias manufactureras a lo largo del intervalo que va de 1994-1999 fue de 8.0.

\subsection{Subsectores con aumento de costos}

En el cuadro 2 se presenta el caso del subsector 37 de la industria manufacturera mexicana que corresponde a las industrias metálicas básicas; contrariamente a los casos anteriores, este presenta aumento efectivo de costos durante el periodo. Este cuadro es análogo al del subsector 33 precedente, salvo en la cuarta columna, pues en ella se muestra la suma acumulada de la reducción real de costos en millones de pesos de 1993.

Las clases industriales del subsector 37 con mayor reducción real de costos son: fundición y/o refinación de metales no ferrosos; laminación, extrusión y/o estiraje de cobre y sus aleaciones, y fundición y/o refinación de cobre y sus aleaciones; la primera de 


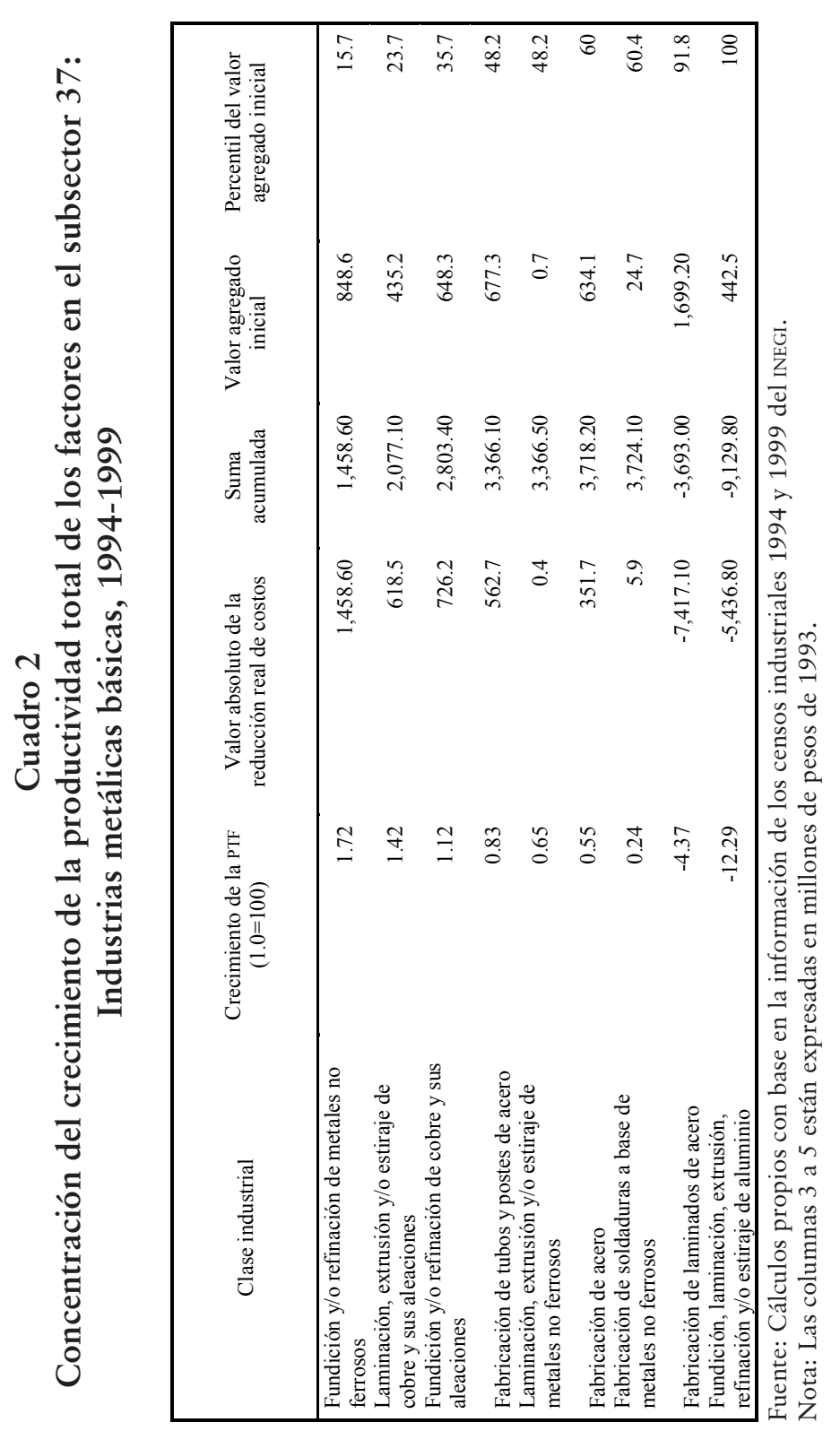


ellas tuvo una reducción absoluta de costos de 1,458.6 millones de pesos de 1993, en tanto que las otras dos actividades industriales reportaron disminuciones de 618.5 y 726.2 millones de pesos, respectivamente, a precios de 1993. De manera contrastante, las clases que presentaron aumento de costos fueron: fundición, laminación, extrusión, refinación y/o estiraje de aluminio y fabricación de laminados de acero.

\section{Gráfica 2}

\section{Subsector 37: Industrias metálicas básicas}

La gráfica 2 muestra en el eje de las ordenadas la suma acumulada 6000 de la reducción real de costos, mientras que el caso previo mos4000 - traba en el mismo eje el percentil de la misma reducción real de costos. Este cambio en la presentación hace que la interpretación

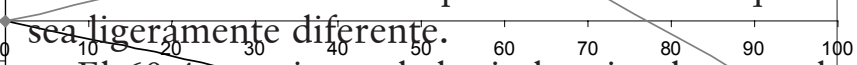
-2000 El 60.4 por ciento de las industrias de este subsector medidas por su valor agregado, reportaron una reducción real de costos, en tanto que el porcentaje restante de las clases industriales tuvie-

ron aumento de los mismos. El promedio ponderado de incre-

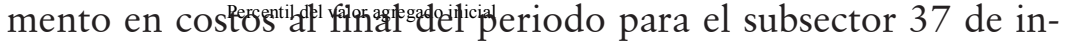
dustrias metálicas básicas fue de 164.08 por ciento.

Como se observa en la gráfica 3 , otros subsectores que también mostraron aumentos de costos fueron: el 31 de alimentos, bebidas y tabaco; 32 producción de textiles, prendas de vestir e industrias del cuero; 34 producción de papel, productos de papel, imprentas y editoriales y el 38 que corresponde a manufacturas de productos metálicos, maquinaria y equipo.

El subsector 31 tuvo 82.4 por ciento de clases industriales con reducción real de costos; el porcentaje de clases industriales con reducción de costos de los subsectores 32, 34 y 38 fue de 34.6, 


\section{Gráfica 3 \\ Subsectores con reducción real de costos}
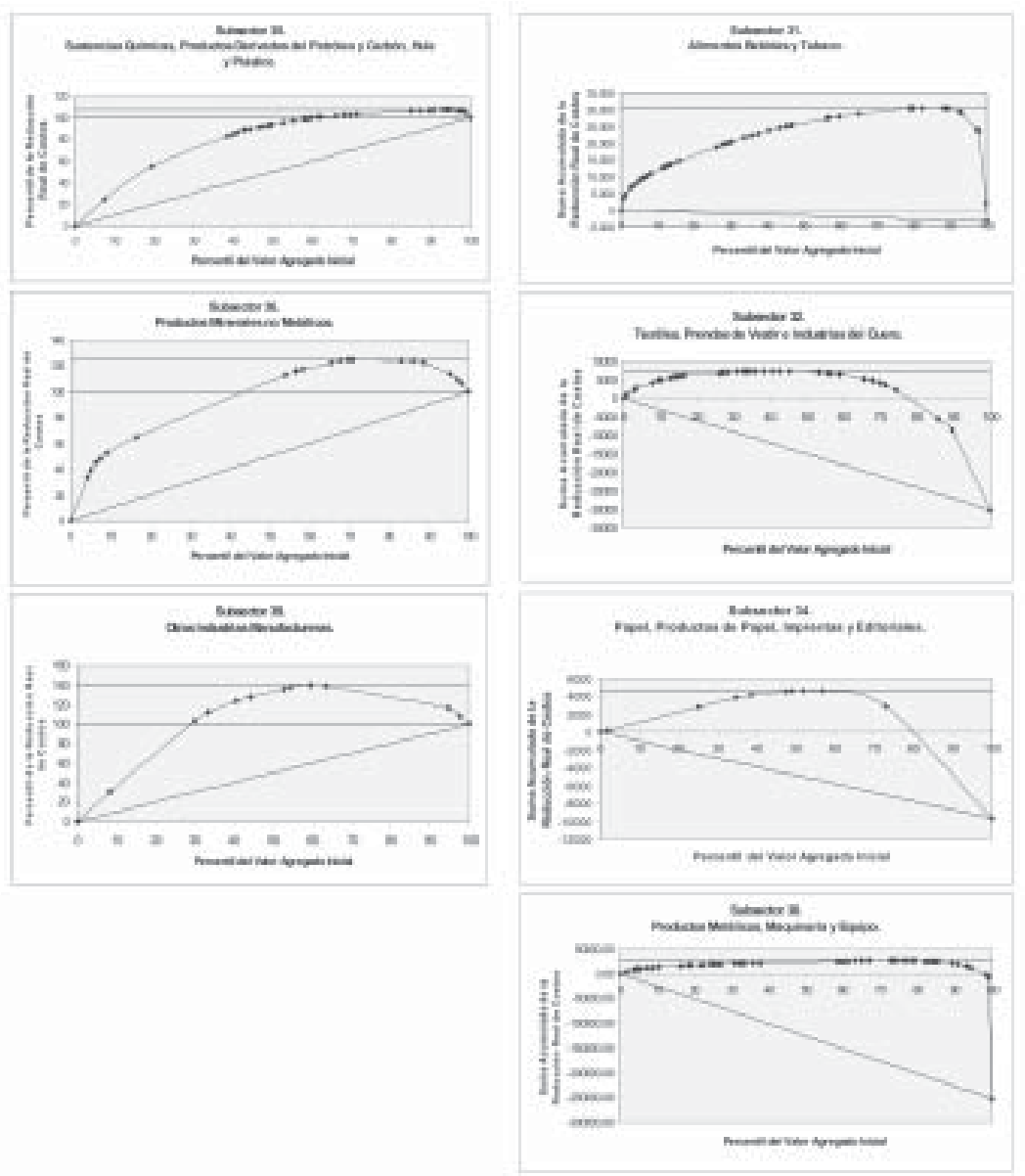

51.9 y 77 por ciento, respectivamente. Los subsectores 31 y 32 presentaron porcentajes promedio ponderados de aumento real de costos de 9.01 y 118.0, respectivamente, al final del periodo. Los porcentajes en promedio ponderado de aumento de costos de los subsectores 34 y 38 fueron los más altos de la industria manufacturera siendo éstos de 334.5 y 544.7 por ciento. 
Las clases industriales con reducción de costos significativa ${ }^{11}$ del subsector 31 fueron: elaboración de concentrados para caldos de carne de res, pollo, pescado, maricos y verduras; congelación y empacado de carne fresca; envasado de té; elaboración de bebidas destiladas de caña; fabricación de grasas y aceites animales; elaboración de cocoa y chocolate de mesa; elaboración de café soluble; elaboración de harinas de maíz; tostado y molienda de café; preparación y envasado de concervas de pescados y mariscos; elaboración de helados y paletas; elaboración de dulces, bombones y confituras; deshidratación de frutas y legumbres; elaboración de almidones, féculas y levaduras; beneficio de arroz; molienda de nixtamal y beneficio de café. En general, todas estas industrias se relacionan con alimentos y bebidas de consumo básico humano, y resulta lógico el que, siendo de consumo generalizado, sus costos tendieran a reducirse mediante la utilización de mejores técnicas productivas o de un uso más eficiente de los recursos.

Contrariamente, las industrias que reportaron incrementos de costos significativos en el subsector 31 fueron: elaboración de otros productos de molino a base de cereales y leguminosas, incluye harinas; elaboración de leche condensada evaporada y en polvo; beneficio de tabaco; elaboración de piloncillo o panela; elaboración de concentrados, jarabes y colorantes naturales para alimentos; fabricación de puros y otros productos de tabaco; elaboración de sopas y guisos preparados. De éstas, las más relevantes en cuanto a valor agregado son: elaboración de concentrados, jarabes y colorantes naturales para alimentos. Otras clases relevantes de este subsector en cuanto a personal ocupado son la elaboración y venta de pan y pasteles, tortillerías, así como la elaboración de refrescos y otras bebidas no alcohólicas, que presentaron una reducción real de costos. Esto es importante dado que, en conjunto, emplean a más de 38 por ciento del personal ocupado en este subsector y más de 8 por ciento del personal ocupado en todo el sector manufacturero.

El subsector 32 tuvo a las siguientes clases industriales con reducción real de costos significativa: fabricación de algodón absorbente, vendas y similares; fabricación de encajes, cintas, etiquetas y otros productos de pasamanería; hilado y tejido de regenerados; fabricación de medias y calcetines; fabricación de calzado de tela con zuela de hule o sintética; fabricación de sombreros,

${ }^{11}$ Una clase industrial tiene un aumento de costos significativo si el crecimiento de la PTF es menor a menos uno. 
gorras y similares, hechas de palma y otras fibras duras; tejido a mano de alfombras y tapetes de fibras blandas; fabricación de fieltro y entretelas de fibras blandas; curtido y acabado de cuero; tejido a máquina de alfombras y tapetes de fibras blandas; fabricación de suéteres; confección de prendas de vestir de cuero, piel y materiales sucedáneos para caballero; y fabricación de fieltro y entretelas de fibras blandas. En este caso, se observa que todas estas clases industriales están vinculadas con prendas de vestir y fibras utilizadas por el ser humano incluyendo cuero y calzado; destacan también las clases que tienen que ver con la fabricación de tapetes y alfombras; dentro de estas clases se encuentra la fabricación de calzado que durante este periodo enfrentó una situación difícil ya que redujo su valor agregado y disminuyó su personal. En este tipo de industrias los costos se redujeron entre 1994 y 1999.

Las industrias del subsector 32 que tuvieron engrosamiento de costos son las siguientes: fabricación de calzado principalmente de cuero; hilado de fibras blandas; confección de otros artículos con materiales textiles naturales o sintéticos; acabado de hilos y telas de fibras blandas; confección de otra ropa interior; fabricación de ropa exterior de punto y otros artículos; fabricación de cordelería de fibras de todo tipo, naturales o químicas; confección de ropa exterior para niños y niñas; fabricación de huaraches, alpargatas y otro tipo de calzado no especificado anteriormente; confección de ropa exterior para dama hecha en serie; confección de guantes, corbatas, pañuelos y similares.

El subsector 34 tuvo solamente una clase industrial con descenso de costos significativo, dedicada a la fabricación de envases de papel. Por otro lado, las industrias que incrementaron costos fueron la fabricación de papel y la impresión y encuadernación.

Finalmente, el subsector 38 tuvo 15 clases de actividad industrial con reducción de costos significativa. La industria que más redujo costos durante el periodo fue la de fabricación y ensamble de carrocerías y remolques para automóviles y camiones; otras que también redujeron sus costos de producción fueron las de fabricación de motores y sus partes para automóviles y camiones, fabricación de equipos para soldar, fabricación y ensamble de motocicletas, bicicletas y similares; fabricación y reparación de utensilios agrícolas y herramientas de mano sin motor; fabricación, ensamble y reparación de equipos eléctricos de ferrocarriles y de aeronaves; fabricación de electrodos de carbón y grafito; fabricación de cintas y discos magnetofónicas, relojes y sus par- 
tes; fabricación y reparación de máquinas fotocopiadoras, motores, etc. Como era de esperarse en este subsector, las industrias más activas experimentaron disminuciones efectivas de costos.

En el subsector 38 las industrias que aumentaron sus costos significativamente fueron: fabricación de hojas de afeitar, cuchillería y similares; fabricación y reparación de embarcaciones; fabricación y reparación de calderas industriales; fabricación de materiales y accesorios eléctricos; fabricación de alambre y productos de alambre; fabricación de filtros para líquidos y gases; fabricación de estructuras metálicas para la construcción; fabricación de partes para el sistema de suspensión de automóviles y camiones; fabricación, ensamble y reparación de equipos y aparatos para comunicación, transmisión y señalización; fabricación de chapas, candados, llaves y similares; fabricación de anteojos, lentes, aparatos e instrumentos ópticos y sus partes; fabricación, ensamble y reparación de maquinaria y equipo para otras industrias específicas.

La clase más importante de este subsector es la fabricación y ensamble de automóviles y camiones, que durante el periodo realizó una reestructuración importante de personal así como inversiones significativas y logró una reducción de costos. Otra de las clases relevantes es la fabricación de partes y accesorios para el sistema eléctrico automotriz que en el periodo obtuvo una ligera reducción de costos con una inversión considerable.

\subsection{Industria manufacturera en su conjunto}

El sector manufacturero en su conjunto presentó aumento real de costos, a pesar de que la mayoría de las clases industriales registraron disminuciones; en la gráfica 4 se observa que 74.5 de las clases industriales experimentaron baja de costos; sin embargo, aquellas industrias que los aumentaron tuvieron un peso muy importante sobre las primeras, determinando el efecto global desfavorable.

Las clases industriales mencionadas en los apartados anteriores que presentaban caída real de costos significativa fueron $76 \mathrm{y}$ representan más de 25 por ciento del total de la industria manufacturera y más de 38 por ciento de las que redujeron costos. En cambio, las clases industriales que tuvieron una ampliación significativa fueron 44 , que representaron poco más de 14 por ciento del total de la industria manufacturera y 46 por ciento del total de las que incrementaron sus costos. 
Gráfica 4

Industria manufacturera en su conjunto

En un análisis más detallado de las clases industriales que tuvieron una mayor reducción real de costos con relación a su costo inicial se observó de manera recurrente que sus resultados se debieron fundamentalmente a un incremento, tanto en términos absolutos como relativos (con respecto al promedio del sector manufacturero), del valor agregado censal bruto. Se puede inferir que estas clases industriales realizaron una reestructuración importante de su personal ocupado ya que éste disminuyó; asimismo, sus activos fijos netos también experimentaron una disminución relativa moderada; por lo tanto puede decirse, de manera general, que con los mismos insumos se produjo más, o alternativamente, con menos insumos se produjo lo mismo.

En el caso de las clases industriales que sufrieron los mayores aumentos de costos con respecto a su costo inicial pueden destacarse algunos aspectos: primero, se advierte que se trata de industrias más grandes en cuanto a valor agregado censal bruto en comparación con las que reportaron las mayores reducciones de costos. En segundo lugar, registraron reducciones importantes en cuanto a sus valores agregados censales y tuvieron un ligero incremento en sus variables de personal ocupado total y de activos fijos netos. En suma, utilizaron sus factores de producción de manera menos eficiente, lo que repercutió en costos reales superiores.

Una de las hipótesis que se ha manejado es que las clases industriales que redujeron más sus costos corresponden a industrias con un mayor dinamismo: una forma de corroborar este dinamismo es observar si las exportaciones en estas industrias fueron mayores y, consecuentemente, su integración al mercado

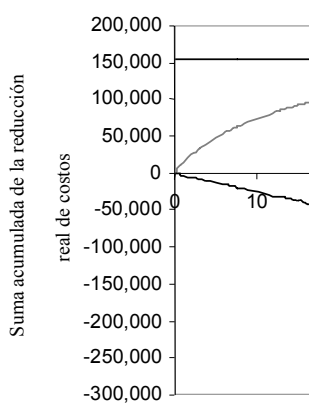


mundial es un elemento que contribuye a explicar sus resultados. Un ejemplo es el subsector 38, en el que se encuentra la clase de fabricación de motores y sus partes para automóviles y camiones de la industria automotriz que presentó reducción real de costos y un incremento significativo en sus exportaciones. De manera similar podemos hablar de la clase dedicada a la confección de prendas de vestir de cuero, piel y materiales sucedáneos para caballero. Con estos ejemplos no se puede concluir que todas aquellas clases industriales que redujeron costos hayan mostrado un mayor dinamismo exportador, ya que corroborar esta hipótesis requiere de una mayor utilización de datos, así como del tratamiento estadístico apropiado.

De todo lo anteriormente expresado se pueden resumir algunos puntos.

i. El crecimiento (o reducción de costos) no ocurre de manera uniforme y generalizada entre las diferentes clases de actividad industrial. Estos resultados son congruentes con los encontrados por Harberger $(1990,1998)$ y por Torre (1996); ambos observan que el crecimiento es un proceso concentrado y heterogéneo. Unas pocas clases industriales explican el 100 por ciento de la reducción real de costos que ocurre al interior del subsector al que pertenecen durante el periodo.

ii. El resto de las clases industriales incluye ganadores y perdedores cuyas contribuciones a la PTF se cancelan unas con otras.

iii. Las clases industriales perdedoras juegan un papel muy relevante, ya que contribuyen con las variaciones observadas en cuanto al desempeño agregado de la PTF.

iv. Asimismo, la forma de las gráficas muestra que la reducción real de costos es dispar y heterogénea entre clases industriales y entre subsectores, acentuándose más en unos en comparación con otros.

v. Los resultados de este estudio se apegan al planteamiento de Harberger (1998), en el sentido de que la reducción de costos se presenta como un proceso de tipo 'hongo' más que de tipo 'levadura'. 


\section{Conclusiones}

Este trabajo analizó la productividad total de los factores como reducción o aumento real de costos en los diferentes subsectores de la industria manufacturera mexicana entre 1994-1999. Los subsectores que experimentaron reducciones de costos fueron el $33,35,36$ y 39; mientras que los sectores restantes mostraron incremento de costos.

Los resultados expuestos confirman la idea de que el proceso de crecimiento ocurre de manera desigual y heterogénea, apegándose más a la visión de un proceso mushrooms (hongos) y no tanto a un proceso yeasts (levadura). La mayor reducción de costos tiende a concentrarse en pocas clases de actividad industrial; otras clases los reducen en menor medida y otras los aumentan; éstas últimas son relevantes al contrarrestar una parte del efecto de las que los reducen al interior de un mismo subsector; adicionalmente, contribuyen con las variaciones observadas en cuanto al desempeño agregado de la PTF en cada subsector y en la industria manufacturera en su conjunto.

La evidencia presentada muestra que en algunos subsectores ocurren las condiciones que favorecen el ahorro de costos entendidos como mejoras en PTF, mientras que en otros ocurre lo contrario. Esto suguiere que en aquellos segmentos de la industria en donde sucedieron aumentos de costos, probablemente el cambio tecnológico y, en general, todos aquellos aspectos que permiten mejorar la productividad, implican un proceso más endeble y que puede ser afectado por condiciones propias de cada industria así como por la incidencia de cambios o choques externos que determinan costos mayores.

Aspectos como las fluctuaciones cíclicas de la demanda, los ciclos de vida de los productos, la orientación hacia el mercado interno o externo, la resistencia hacia la adopción de nueva tecnología, la heterogeneidad en cuanto al uso de los factores de producción, diferencias de estructura industrial, evolución, grado de integración con otras empresas e industrias, redes de interacción e intercambio intra e interindustrial, localización y hasta las políticas públicas que se aplican en cada clase industrial son elementos que causan efectos que podrían estar manifestándose bajo la forma de aumentos o reducciones de costos en el periodo abarcado en este estudio.

De la misma manera, puede decirse que los movimientos en costos pueden suponer mejoras en el know how técnico que pue- 
de deberse a una mejor estructura organizacional puesta en marcha en la empresa, uso de equipo nuevo, mejores habilidades de los trabajadores, etc. Interviene también la forma en que se difunden las nuevas técnicas de producción, que frecuentemente varían entre empresas e industrias y se sujeta a las condiciones económicas que conforman el entorno en el que se desenvuelven ambas. En general, las empresas propensas a introducir innovaciones lo hacen esperando obtener mayores beneficios, reducir costos, reemplazar maquinaria y equipo, y ofrecer mejores precios al consumidor.

Los estándares de habilidades (educación, experiencia, etc), técnicas productivas, barreras de entrada, patentes, localización geográfica, inercia e incertidumbres son relativamente comunes para empresas de una misma industria. En términos generales, las posibilidades de reducir o aumentar costos reales suelen afectar de manera semejante a firmas que enfrentan un contexto similar. En este sentido, el aumento o reducción de costos puede estar sujeto a la capacidad de adaptación de las empresas y a su disposición de aprender bajo un escenario dinámico y cambiante.

Los resultados presentados aquí de ninguna manera son concluyentes y sólo contribuyen a mostrar evidencia empírica sobre aumentos o reducciones de costos en un período reciente, muy específico de la evolución de la industria manufacturera mexicana.

La agenda de investigación en la lista de temas por analizar en futuros trabajos incluye, primero, la explicación y causalidad tanto de la baja en costos como del aumento de los mismos, profundizando en aspectos más individualizados de cada subsector y clase industrial, investigando por ejemplo: si las industrias que abatieron costos son aquellas que realizaron mayores exportaciones a lo largo del periodo, si realizaron inversiones en activos, maquinaria y equipo, es decir, si adoptaron tecnología, efectuaron innovaciones, patentaron o si realizaron actividades de investigación y desarrollo, etc., y alternativamente si la explicación se debe al entorno institucional, organizacional o a otros aspectos. También sería interesante contrastar estos resultados con los que podrían obtenerse con otras formas de medición de la PTF que se basan en la aplicación del análisis de frontera. 


\section{Bibliografía}

Griliches, Zvi (1996), “The Discovery of the Residual: A Historical Note", Journal of Economic Literature, vol. 34, núm. 3, septiembre., pp. 1324-1330.

Grether, Jean-Marie (1999), "Determinants of technological diffusion in mexican manufacturing: a plant-level analysis", World development, vol. 27, núm. 7, Great Britain, pp. 1287-1298.

Harberger, Arnold C. (1990), Reflections on the Growth Process, Working Paper, World Bank.

(1998), "A Vision of the Growth Process", The American Economic Review, vol. 88, Issue 1, mar., pp. 1-32.

Hulten, Charles R., (2000), Total Factor Productivity: A Short Biography, National Bureau of Economic Reserarch, working paper 7471, Cambridge, Massachusetts, enero, pp. 75 .

López-Acevedo, Gladys (2002a), Tecnology and Firm Performance in Mexico, JEL codes: L60, L20, J31, J38, World Bank, pp. 1-35.

(2002b), Technology and Skill Demand in Mexico, JEL codes: L60, L20, J31, J38, World Bank, pp. 1-21.

- (2002c), Determinants of Technology Adoption in Mexico, JEL codes: L60, L20, J31, J38, World Bank, pp. 1-39.

Meza G., Liliana y Ana Belén Mora (2002), RひoD intensity in mexican manufacturing firms, World Bank.

Nadiri, Ishaq M. (1970), "Some Approches to the Theory and Measurement of Total Factor Productivity: A Survey", Journal of Economic Literature, vol. 8, núm. 4, pp. 11371177. 
OECD (2001a), Science, Technology and Industry Outlook. Drivers of Growth: Information Technology, Innovation and Entrepreneurship, special edition, Paris.

(2001b), OECD Productivity Manual: A Guide to the Measurement of Industry-Level and Aggregate Productivity Growth, Paris.

Pablo M., Federico (1997), La Concentración Industrial en la Economía Española: 1980-1992, Universidad de Alcalá, España.

Prescott, Edward C. (1998), "Lawrence R. Klein Lecture 1997: A Theory of Total Factor Productivity", International Economic Review, vol. 39, núm. 3, pp. 525-551.

Sargent, Timothy C. y Edgard R. Rodríguez (2000), "Labour or Total Factor Productivity: Do We Need to Choose?”, International Productivity Monitor, 0 (1), pp. 41-44, documento web <http://www.csls.ca $>$

Solow, Robert M. (1957), "Technical Change and the Aggregate Production Function”, The Review of Economics and Statistics, vol. 39, Issue 3, pp. 312-320.

Torre, Leonardo (1996), “Crecimiento Económico y Reducción en Costos Reales: Evidencia de la Industria Manufacturera Mexicana, 1984-90”, Entorno Económico, vol. Xxxiv, núm. 203, agosto, CIE, UANL, pp. 1-4.

World Bank (1998), México: enhancing factor productivity growth, Country economic memorandum, report núm. 17392-ME, pp. 190.

Enviado: 6 octubre del 2003.

Reenviado: 18 de febrero del 2004. Aprobado para su publicación: 19 de marzo del 2004. 